\title{
Tumour Vaccines, Monoclonals, Proteins or Whole Cell Therapies
}

\section{Shahe Boghossian* and Alexei Von-Delwig}

Surgeon, NHS, General and Vascular Surgery, 1294 Warwick Road, Birmingham, West Midlands, B27 6PL, UK

\begin{abstract}
Immunotherapy has dramatically changed the outcome of many tumours. Whether it is by using antibodies as a humoral form of tumour control and clearance or active vaccines that allow the immune system to mount a response, the methodologies are quite different yet sharing an inherent similarity; finding the right epitope and eliciting the right response within the proper time frame of cellular maturity. The initial enthusiasm of using degraded proteins and priming T-cells against these vaccines has faded away with less than satisfactory outcomes in clinical trials. Albeit our understanding of how Dendritic Cells and T-cells interact and display antigens has been improving, we have not been able to clearly pinpoint a proper way in using peptide vaccines in clinical settings. This eventually prompted the use of whole proteins, necessitating antigen processing inside antigen presenting cells to give to suitable immunogenic epitopes. This has proven successful in a few clinical trials so far; however, recent evidence clearly demonstrates that eradication of a tumour is not just a matter of which protein is most likely to be immunogenic, but a combination of several other factors in its immediate and possibly distant environment. Hence our methodology in tumour clearance by using active vaccines is unlikely to be met with much success until we clearly understand the environment which allows these tumours to be nurtured. Henceforth, a whole cell vaccine in the context of enhanced adoptive T- cell immunotherapy will most likely be successful but still will not be the ultimate way in eradicating tumours. Our discussion will focus on several treatment modalities and success stories as well as possible modalities that will prove to be successful in the not so distant future.
\end{abstract}

\section{Introduction}

The latest advancements in tumour immunology have opened new horizons in cancer vaccines. These developments have incorporated crucial inter-cellular signal transduction mechanisms and cellular maturity signals. The clinically successful vaccines that we have incorporated in our clinical practice have come a long way from the early nineteenth century vaccines. With more developments coming to fruition, cancer vaccines in all forms and modalities are likely to complement clinical chemotherapy and surgery with even more success.

\section{Methods}

We ran a search on Pubmed Central, Scirrus, Cancer immunity, Medscape and Cancer-testis antigens dating from 1995-2012 inclusive. We had more than 400 clinical reports and articles of agents, biochemicals, peptides, proteins and whole cell vaccines with or without adjuvants. We filtered these down to agents that showed clinical promise based on its repetitive inclusion in several "Entrez"s or ones that have passed phase I clinical trial . However, any agent that was tested and deemed to be a failure was discarded.

\section{Results}

Several agents have surfaced as key mediators in immunotherapy; two key monoclonal antibodies, Ipilimumab and Tremelimumab have shown a significant advantage in tumour therapy. Another set of proteins and their associated breakdown peptides have shown to have promising results as well. Of particular importance is Sipuleucel-T, which is the first fully approved vaccine against metastatic castration resistant prostate cancer. Additionally our search demonstrated another important modality in controlling tumour progression; the peritumoural environment, the key player being Myeloid Derived Stem Cells. Finally, Listeria Monocytogenes appeared as a plausible answer to some vaccine adjuvants and its use could change the practice of tumour vaccines in the future.

\section{Conclusion}

Tumour vaccines are an important modality in tumour control.
Albeit the fact that they are unlikely to change the surgical management of tumours, they are and will definitely become an important adjunct to tumour growth control, particularly for chemotherapy resistant tumours like pancreas, lung and melanomas. The latest mediocre successes of tumour vaccines will pave the way for more successful vaccines that might ultimately affect the overall survival of tumours.

\section{The Immune Synapse}

Tumour peptides shed by tumours are regarded as exogenous byproducts; they are phagocytosed by Antigen Presenting Cells (APCs), in particular by dendritic cells (DCs) in the vicinity of the tumour. These cells will eventually uptake this debris and present them to $\mathrm{T}$ cells in draining lymphatic nodes. In the draining lymph nodes of the tumour bed lays the most important cellular mechanism of tumour immunology which is the immune synapse [1]. The synapse; which is the interaction of APC/DC loaded with tumour peptide and presented to T-cell clone heralds the start of this crucial interaction. In classic terms, an APC will present antigen on its surface bound either on MHCI or MHCII, its corresponding ligand would be a T- Cell Receptor (TCR) expressed on the surface of CD8 or CD4 T cells respectively. Within this context of immune synapse additional co-stimulatory ligands would be also interacting [2]. In structural terms, the immune synapse is a circular zone of molecular interaction between a $\mathrm{T}$ cell and an APC, with the antigen sandwiched between the TCR-CD4/8-MHC

${ }^{*}$ Corresponding author: Shahe Boghossian, Surgeon, NHS, General and Vascular Surgery, 1294 Warwick Road, Birmingham, West Midlands, B27 6PL, UK, Tel: +447533496375; E-mail: shaboghru@gmail.com

Received January 25, 2012; Accepted May 25, 2012; Published May 27, 2012

Citation: Boghossian S, Von-Delwig A (2012) Tumour Vaccines, Monoclonals, Proteins or Whole Cell Therapies. J Vaccines Vaccin S1:003. doi:10.4172/2157-7560.S1-003

Copyright: () 2012 Boghossian S, et al. This is an open-access article distributed under the terms of the Creative Commons Attribution License, which permits unrestricted use, distribution, and reproduction in any medium, provided the original author and source are credited. 
Citation: Boghossian S, Von-Delwig A (2012) Tumour Vaccines, Monoclonals, Proteins or Whole Cell Therapies. J Vaccines Vaccin S1:003. doi:10.4172/2157-7560.S1-003

complexes, followed by a circumferential zone of molecular interactions. These interactions play a key role in directing the immune response towards either tolerance or immunity against the target protein. Within this context, the interaction of CD80/86 on APCs with CTLA4 an inhibitory protein on $\mathrm{T}$ cells will abrogate the immune response and hence render them intolerant. However the interaction of CD80/86 with CD28- a stimulatory molecule, will result in the development of $\mathrm{T}$ cell immune response against the target antigen [3].

A step further in detail regarding immune synapse is the mechanism of antigen presentation which has undergone immense scrutiny. Antigen Presenting Cells (APC) has mainly two ways of expressing antigens to the immune system. 1) Endogenous antigens, such as intracellular pathogens are usually degraded by a specialised organellethe proteasome. Proteasomes act as an intracellular breakdown organelles whereby all debris mainly proteins will eventually be broken down to 7 or 8 mer peptides and latched on to MHC I molecules for subsequent presentation to TCR/CD8 expressing T cells in draining lymph nodes [4]. 2) Exogenous peptides; such as tumours and viruses are broken down by protease to be latched on to MHC II which would further present it to TCR/CD4 expressing T-cells.

An alternative mechanism has developed within the immune system whereby exogenous peptides rather than being presented with MHCII are expressed on MHC I molecules on dendritic cells and thus interact with CD8 expressing T cells. This mechanism has been termed cross-presentation. It is unclear why this mechanism has evolved when there are clearly two separate modalities of antigen presentation. A suitable explanation would be to offload the incoming traffic of proteins and use a more robust MHC I system which would degrade proteins in a more effective way via the proteasome. Additionally, it would be an ideal mechanism to prime two cells lines CD4, as well as cytotoxic CD8 cells which would be useful in mounting an immune response (Figure 1) [5]. Within these interactions, three elements have come to play a key role in tumour peptide presentation; these are interferon, hyperthermia, and Heat Shock Proteins

\section{Interferon, Hyperthermia and Heat Shock Proteins}

As discussed earlier, the simple display of tumour peptides on MHC-I or II molecules is far from being a simple mechanism of antigen presentation. In fact recent modulation of antigen presentation by several factors have shown that the effectiveness of the presentation of the peptides can be augmented through the use of certain cytokines, such as GM-CSF as well as Interferon $-\alpha$ particularly when dealing with dendritic cell vaccines. Upon stimulation with Interferon- $\alpha$ several intracellular proteins which play a key role in loading peptides on to MHC I have been shown to be increased, in particular TAP1/2 and tapasin. Additionally, under these conditions, the proteasome displays more enhanced activity generating a broad repertoire of 8-9 amino acid peptides to be loaded on MHCs [6]. An additional yet quite controversial effect comes from hyperthermia which is a well established modality in patients with peripheral melanomas. Hyperthermia obviously causes protein denaturation and heralds cellular apoptosis. However, hyperthermia releases Heat Shock Proteins which are on one hand key elements in protecting the cellular proteins from degradation, and on the other hand act as chaperones for the same proteins to be degraded by proteasomes [7]. This duality of their action has prompted many researchers to look into Heat Shock Proteins as modulators in immunotherapy. Two particular candidates have been revealed -HSP70 and HSP90, each having a different mechanism of action. Latest research demonstrates that HSP70 has an important role in chaperoning tumour proteins towards degradation by proteasomes and an equally important role in mounting the degraded products on APCs [8]. Experimental tumour vaccines laden with Mucin1 and HSP70 gene have demonstrated this effect quite dramatically [9]. On the other hand, HSP90 is a chaperone which catalyses the activation and maturation of several proteins involved in proliferation, cell cycle control and anti-apoptosis activity. HSP90 inhibitors in this case act

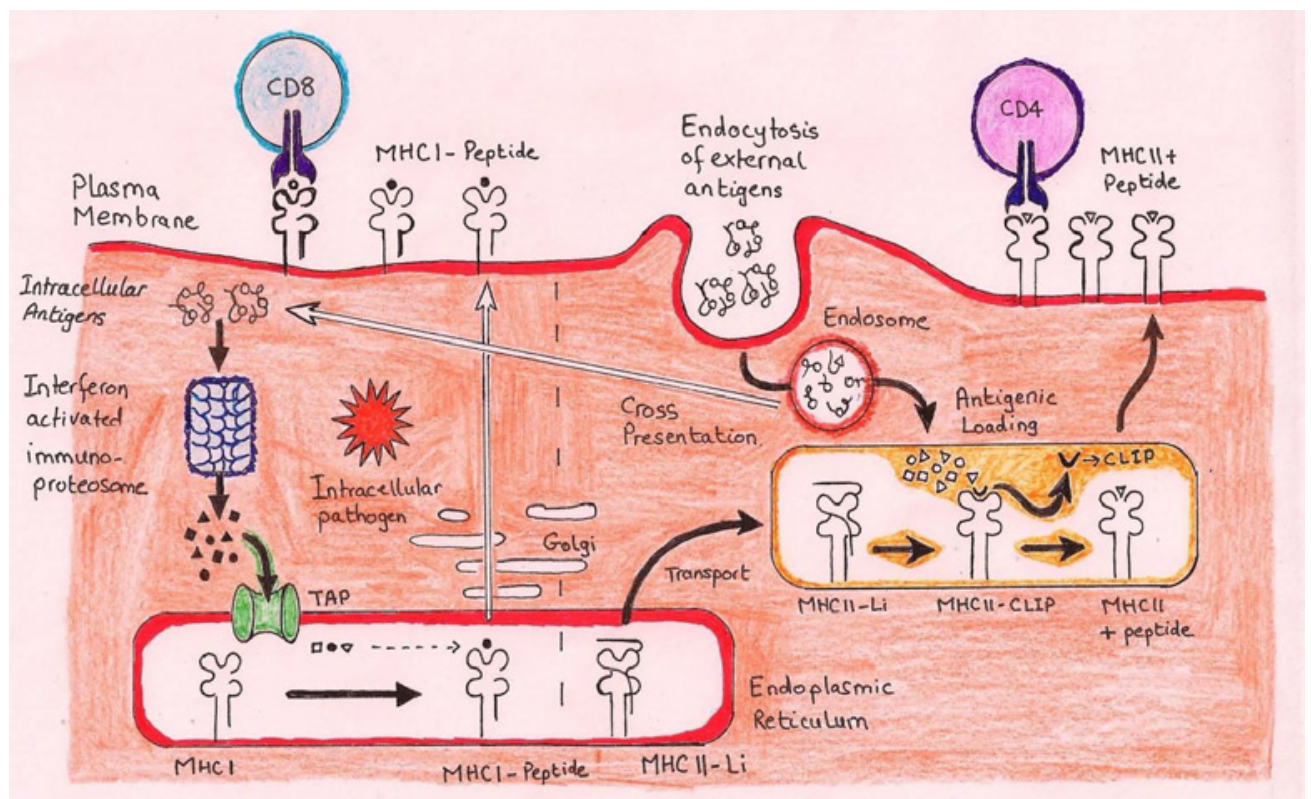

Figure 1: An artist's impression of endogenous and exogenous antigen uptake and presentation. The section on the left displays antigens broken down and display to $\mathrm{MHCl} / \mathrm{TCR} / \mathrm{CD} 8+$ pathway while the right side shows antigens endocytosed and presented to MHCII/TCR/CD4+ pathway. In the middle lies a key pathway called cross-presentation which takes antigens from $\mathrm{MHCll}$ to $\mathrm{MHCl}$ display which is a robust mechanism for presenting tumour antigens as well. 
as a potent adjuvant in any chemotherapy cycle [10]. Several HSP90 inhibitors are in phase I/III clinical trials and the results have been quite promising.

\section{Avidity and Clonal Deletion}

Human immune system has a unique capacity in removing self reactive $\mathrm{T}$ and $\mathrm{B}$ cells in an attempt to prevent autoimmunity. Central deletion occurs in the thymus; the development of high avidity autoimmune cytotoxic $\mathrm{T}$ lymphocytes is promptly deleted during embryological development and as far as early years of neonatal life. An example of "foreign antigen-central tolerance" would be the lack of immune response in neonates with chronic hepatitis B; whereby exposure to the antigen in utero in a manner that it is regarded as "self antigen" results in the persistence of infection postnatally. In the process of negative selection the entire repertoire of self proteins is screened and the most reactive $\mathrm{T}$ cells are selected towards apoptotic deletion. A small subpopulation of low affinity $\mathrm{T}$ cells escape to the peripheral lymph nodes were those cells play a crucial role in autoimmunity; or in a diametrically opposite manner can be used to mount response against tumours. The relative maturity of the DCs involved in the antigen presentation, as well as the expression of costimulatory signals plays an additional role in the ultimate outcome of the immune response.

a)

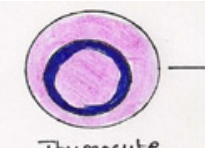
Thymocyte b)
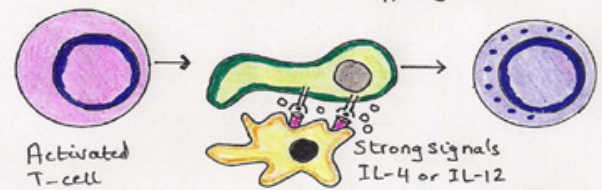

TH1 or TH2

c)
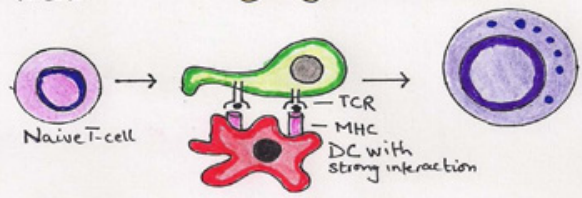
Activated

d)
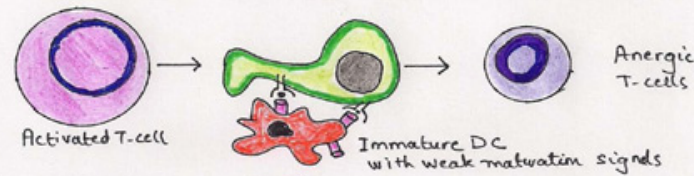

e)
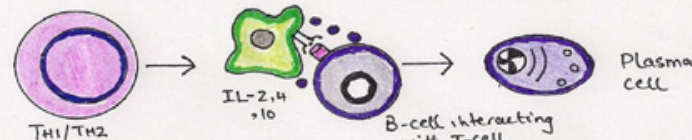

f)
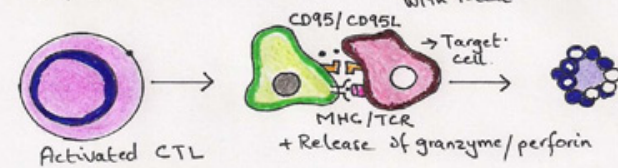

Figure2: an artist's impression of 6 ways of T-cell maturity or regression. a) Central deletion of auto-reactive in thymus. b) Strong stimulation of lymphocytes by dendritic cells and their subsequent development towards comitogenic $\mathrm{TH} 1$ or $\mathrm{TH} 2$. c) A naive non-stimulated cell maturing with strong cells to become activated. d) The same activated T-cell becomes anergic when it does not receive enough stimulation. e) Th1 or Th2 allowing the transformation of B cells to plasma cell. f) Lysis of target cell with strong interaction and the costimulation of death receptor CD95/95L.
Evidence is mounting that indeed the Tumour Infiltrating Lymphocytes (TIL) possesses the necessary avidity and reactivity to mount an immune response against tumours; however, the peripheral tolerance mechanisms that are effectively dampening this activity seems to reside more in the peritumoural environment [11]. The dose, duration and site (subcutaneous, intratumoural or intravenous) of vaccine delivery ultimately determines the modality of the immune response (Figure 2) [12]. Ex vivo peptide loading on DC works in some settings, even when injected directly into tumours but the novel discoveries in peripheral tolerance have shifted the attention from the classical scenario of Dendritic Cell-Tumour Antigen-T cell towards two rather important cells; Regulatory T cells, Tregs and Myeloid Derived Stem Cells which we will be discussing further on [13].

\section{Monoclonals}

Obviously the discussion about monoclonal antibodies will not be exhaustive but we will keep the discussion relevant to monoclonal antibodies involved inter-cellular signal transduction between T-Cell and APC only.

Two monoclonal antibodies Ipilimumab and Tremelimumab against CTLA4 played a key role in immunotherapy against solid tumours as the development of another novel monoclonal antibody.

Ipilimumab (IgG1) is a fully human monoclonal antibody patented by Bristol Myers Squibb ${ }^{\circ}$. It blocks the interaction of CTLA4 with CD80/86 causing an abrogation of the immune response in the T-cell population or total dampening of the immune synapse. A similar mechanism of action has been described for Tremelimumab (IgG2). Currently there are at least 25 registered clinical trials which have been initiated involving Ipilimumab with various combinations against solid tumours mainly against melanoma, metastatic castration resistant prostate carcinoma (MCRCP) and lung carcinoma. A recent trial by Robert $\mathrm{C}$ et al in patients with previously untreated metastatic melanoma, 502 patients were randomly assigned to receive Ipilimumab $10 \mathrm{mg} / \mathrm{kg}$ plus dacarbazine $850 \mathrm{mg} / \mathrm{m}^{2}$ or dacarbazine plus placebo. In a cardinal objective of overall survival (OS), the Ipilimumab+Dacarbazine had a $47.3 \%$ survival versus $36.3 \%$ for the dacarbazine only group at 1 year follow-up. The advantage continued after 3 years with $20.8 \%$ for the combined therapy versus $12.2 \%$ for the dacarbazine only group [14]. A key point in this trial was the fact that none of the patients were screened for the BRAF mutation-V600E. A similar successful result has been observed with metastatic castration resistant prostate cancer but the results have not been made available yet. Tremelimumab, which has been developed after Ipilimumab has been undergoing phase II clinical trials on patients with refractory colorectal cancer but there are no phase III randomised clinical trials have been reported yet [15].

On the other hand, PD1/PD1L is a similar molecule to CTLA4 and shares almost the same mechanistic interaction between T-cell and DCs whereby it interacts as potentiator of inhibitory signals causing the activation of the T cells upon antigen loading. BMS 963558 is an IgG4 antibody which interacts PD1 blocking its interaction with PDL1. At the moment there are a few pilot studies of this novel immunotherapeutic agent, with studies of its efficacy being used in lung cancer, renal cancer melanoma and colorectal cancer [16]. Although these "bulleted" therapies do not conform to the classical vaccination modality; however, a structurally homologous diabody or scFv body specific to CTLA-4 might act as an important adjunct to vaccine therapy in the future. Similarly, it is possible to construct a non-signalling CTLA-4 decoy agent through gene therapy which would bind to CD86 co stimulatory 
molecule, but would still activate the immune response; however, these modalities are still in very early stages of development.

\section{The candidate peptides and vaccine trials}

Peptide vaccines have been the foundation of tumour vaccines loaded onto dendritic cells. The discussion will focus on common Tumour Associated Antigens in common tumours and their corresponding clinical trials

Carcinoembryonic Antigen: Carcinoembryonic Antigen (CEA) is a tumour associated antigen secreted in almost all gastrointestinal tumours of endodermal origin but more commonly in lower gastrointestinal tumours. CEA is a glycoprotein that is elevated in familial and non-familial colorectal carcinomas. Colorectal cancer being a common tumour; CEA was the first tumour associated antigen to be cloned. Several CEA splice variants have undergone extensive trials in mice with very encouraging results showing an elevation of Cytotoxic CD8+ T cells as well as increase in the secretion of interferon. Unfortunately, the same translational analogy was not observed in human trials albeit the fact that Phase I/II trials have been successfully completed with a substantial immune response but mediocre clinical response. To overcome the resistance, the CEA gene has been cloned with TRICOM (Triad of Co-Stimulatory molecules; B7.1, LFA3 and ICAM-1) or cloning the CEA gene with GM-CSF or IL-4 which has demonstrated a better efficacy and safety profile $[17,18]$. Depending on the epitope or splice variant used with the corresponding HLArestricted $\mathrm{T}$ cell, many of the murine or even human trials showed evidence of development of autoimmunity in the form of colitis, and some cases even requiring cessation of vaccination. To overcome the lack of clinical response one of the early pioneering trials by Gulley JL et al incorporated MUC1 antigen with CEA and TRICOM which clearly demonstrated a sustainable and durable response [19]. With all the multitude of murine trials, only a handful of trials have made any clinically significant impact; however, the combinatorial use of two antigens with co-stimulatory molecules holds promise for the future.

MAGE: MAGE gene family is over expressed in a variety of tumours but not in normal tissues except testes hence it is described as a cancer testis antigen. MAGE3 is overexpressed in Non-Small Cell Lung Carcinoma (NSCLC) and Melanoma. Its vaccine properties have been the focus of attention for vaccine trials. Unfortunately, as with other vaccine trials against melanomas, there have been dramatic success stories but equally disappointing results as well. One of the earliest phase I trials was conducted by Banchereau et al., the authors vaccinated 6 patients with a spectrum of melanoma antigens with Keyhole Limpet Hemocyanin (KLH) loaded on to CD34+ DC which resulted in no significant advantages in survival in patients with stage IV melanoma albeit the fact that 4 of the patients were still alive as of the assessment date [20]. Another successive phase II trial was attempted by a different team when 56 patients with stage IV melanoma were vaccinated with melanoma antigens including MAGE3, 28 of the participants were able to complete the trial. Six of the patients were disease free for more than 5 years with an elevation of interferon levels against the antigens in another 3 patients. Vitilgo was a side effect of the treatment but was not a factor of prognosis or tumour clearance [21]. Another recent phase II trial randomised 2 groups to receive MAGE, Tyrosinase 1, gp100 and MART with or without low dose IL-2 OR, autologous tumour lysate with or without IL-2 in patients with disseminated melanoma. Median survival for all patients was 18.5 months with no difference between the subgroups; however, there were 2 partial responses and 3 patients with stable disease in the 9 patients receiving peptide+IL-2. However, the costs of treatment compared with the mediocre results were not enough to justify further treatment [22]. A dramatic change in approach towards MAGE3 was pioneered with landmark MAGRIT (MAGE-A3 as Adjuvant Non Small Cell Lung Cancer Immunotherapy) trial. The trial started recruiting participants in 2007 based on previous promising phase II results which demonstrated a $27 \%$ reduction in lung cancer recurrence compared to surgery alone. The trial is aiming to recruit 2270 patients with IB/II/III stage NSCLC, from 580 centres in 33 countries with a $2: 1$ randomisation of patients receiving either immunotherapy or placebo. The vaccine is composed of purified recombinant MAGE-A3 protein fused to protein D and a special adjuvant. Thirteen injections will be given intramuscularly over 27 months and the patients will be followed up for 5 years with the primary endpoint being disease free survival. As of this moment there are more than 1600 patients already randomised and the trial will finish accrual by the end of 2011, the results of which are keenly awaited [23] .

MUC1: MUC1 is a member of the mucin family of transmembrane glycoproteins found on almost all apical cell surfaces in normal noncancerous cells. The protein undergoes extensive O-glycosylation and acquires a highly branched structure. Overexpression, defective glycosylation or diffuse expression on the basal and apical surfaces is associated with carcinogenesis. Structurally, MUC1 is a transmembrane glycoprotein with an intracellular portion involved in cell signalling. The extracellular portion has a variable number of tandem repeats (VNTR) of 20 amino acid residues, rich in serine, threonine and proline permitting O-glycosylation. Functionally, MUC1 has a multitude of roles that are either directly or indirectly involved in preventing cell death, shielding the tumour cell from chemotherapy and $\mathrm{T}$ cells, promoting cell growth through an EGFR dependent manner and promoting tumour cell invasion through the stabilisation of beta-catenin. MUC1 is further regulated by hormones like estrogens (breast cancer), insulin and growth hormone [24]. On the other hand, structural variabilities between the nascent and the defectively glycosylated MUC1 could open potential sites for glycosylated peptide vaccines in patients with breast, pancreas, lung, ovarian and colon carcinomas, or in other terms almost all tumours with luminal epithelium. Early vaccines of MUC1 antigen used peptide structures only but these have been further superseded with GlaNAc tagged peptide vaccines [25]. An alternative model has been implemented with the use of the entire MUC1 gene and IL-2 (TG4010) in patients with stage III/IV NSCLC which gave good results in phase II clinical trial [26]. However, the pivotal trial comes from a new agent called Emepepimut (L-BLP25) in patients with nonresectable stage III NSCLC. The vaccine mimics the core of the MUC1 and has been showing promising results in phase II trials with 1 year survival approaching 82\% and 2 year survival approaching 64\% [27]. The phase III START clinical trial which has randomised 1444 patients so far is finishing accrual in June 2011; the results of which are keenly awaited.

Heparanase: As the name implies is the key enzyme that breaks extracellular and intracellular heparin sulphate to oligosaccharides. Heparanase plays a key role in tumour metastasis with a direct causality between its expression and tumour invasiveness. Heparanase makes an ideal candidate for immunotherapy because of its universal expression in metastasis prone tumours [28]. Heparanase is expressed in sites of inflammation and highly expressed in liver tumours, pheochromocytomas, gastric tumours, sarcomas as well as a multitude of other tumours. Early experimental design of vaccines against heparanase aimed at using microRNA against heparanase in metastatic breast carcinoma showed encouraging results [29]. Subsequent vaccines using HLA-A2 restricted CTLs showed further encouraging results, due to the use of branched multiantigenic peptides which gave a 
broader spectrum of action, high specificity and high safety [30]. There have been no randomised clinical trials with heparanase; however, we can say with certainty that there are high hopes for a vaccine against heparanase.

Survivin: Survivin is regarded as a major player in tumourigenesis. Survivin is an inhibitor of apoptosis; it inhibits caspase 3 and 7 and is regarded as an oncogene. During embryogenesis, Survivin is highly active to promote growth and proliferation of the entire organism. The protein product of human Survivin is usually absent in life except under severe inflammatory conditions and obviously in tumours. Survivin has been found to be expressed in almost all tumours subtypes and its expression is regarded as a poor prognostic marker. Survivin promotes neural development, hematopoiesis, cardiogenesis but above all in vasculogenesis. Early experimental phase I results with, HLA-A24 restricted, 9-peptide splice variant (survivin-2B80-88) were quite encouraging with potent CTL response against several tumours [31]. The first case report of using Survivin vaccine came from a 72 year old patient suffering from metastatic pancreatic cancer refractory to gemcitabine who initially maintained a partial response that soon became a complete remission after 8 months of vaccine therapy. The patient went back into progression after weaning off from vaccination [32]. In a small phase I trial on 21 patients with metastatic melanoma, showed a decrease in FOXP3/CD4+/CD25+ regulatory $\mathrm{T}$ cells and myeloid derived stem cells; but there was no statistical clinical significance in terms of tumour progression or durability of response [33]. A recent phase I/II trial in 36 patients with malignant melanoma receiving Survivin peptide, p53 with IL-2 and interferon $2 \mathrm{~b}$, showed a longer mean survival time in patients with stable disease versus patients with progressive disease (18.5 months vs. 5 months). Although the overall number of partial responders was small $(n=11)$, the response rate of almost $24 \%$, indicates a significant response to vaccine treatment [34]. The poor response rate of tumours expressing Survivin especially in chemotherapy resistant tumours makes this antigen a very attractive target for immunotherapy. However, more dynamic approaches in the future are to be expected to tackle such resistant tumours.

Cancer mucosa antigens: Some areas of the body are regarded as immunoprivileged or shielded from the immune system. Recent evidence showed that the expression of intra-luminal antigens could be particularly attractive as immunotherapeutic targets. The intestinal luminal barrier which protects against intestinal bacteria creates a delicate balance between luminal breakdown by macrophages versus luminal invasion by enteric bacteria. A conspicuous antigen among them is Guanylyl Cyclase C (GCC) which is normally expressed endoluminally on the intestinal epithelium and in colorectal tumours [35]. As such, this antigen which we postulate, has been shielded from the immune system should be able to mount a potent immune response since the immune system will regard it as a novel antigen but with limited autoimmune injury. GCC immunisation has been successfully attempted in mice but not in human candidates yet [36]. However, it opens a new dimension in vaccination strategies and may hold much promise for the future.

Glypican 3: The Glypicans are one of the cell surface glycoproteins composed of heparan sulphate with a glycosylphosphatidylinositol membrane anchoring portion. The Glypicans are involved in cell transformation and cell signalling. Elevated levels of Glypican 3 have been reported in Hepatocellular carcinoma, ovarian carcinoma, mesothelioma and thyroid tumours as well as elevated level of Glypican 1 in breast and brain tumours. The expression of the glypicans is confined to the cell membrane with close association of these structures with growth factors and hormones. The glypicans are hereby described as oncofetal antigens just like alphafetoprotein whereby their expression is increased during hepatic embryogenesis, post fulminant hepatitis regeneration focii and tumourigenesis, with specific increase of Glypican 3 in yolk sac derived tumours although to some extent this is not uniquely consistent [37]. Hepatocellular carcinoma being one of the most resistant tumours against chemotherapy has been the focus of Glypican 3 in this particular setting [38]. There are no human phase III trials with Glypican 3 currently but a study of 14 human subjects with HLA-A2 restricted CTLs clones, has demonstrated good immunological response but a mediocre clinical response [39]. Certainly, this novel oncofetal antigen might hold good promises for the future; however, until we get sustained clinical results the success about this new antigen will be confined to the laboratory.

Sipuleucel-T: Sipuleucel has been hailed as a success of immunotherapy. Sipuleucel-T (APC8015) Provenge ${ }^{\bullet}$ is the first therapeutic vaccine for metastatic castration resistant prostate cancer (MCRPC), fast tracked by the US FDA and approved in April 2010. Albeit the fact that the overall survival benefit compared with placebo is 4 months, it principally proves an important concept in immunotherapy [40]. Sipuleucel-T has to be prepared individually for every patient. The patient's blood is withdrawn and the white cells are harvested with specific emphasis on DCs which can be isolated ex-vivo by magnetic beads. These cells are further incubated with a fusion protein comprising of Prostatic Acid Phosphatase- an immunogen or cancer antigen and GM-CSF which acts as an amplifier of the antigenic stimulus; in this case Prostatic Acid Phosphatase. Following an incubation period of 36-44 hours, modulated DCs are infused back to the patient [41]. The combination of Sipuleucel-T with docetaxel confirmed a survival advantage of 4 months which unequivocally proves an advantage of immunotherapy with standard chemotherapy although the cost of treatment is quite significant $(93,000 \$$ per treatment).

Lucanix: Lucanix ${ }^{\text {ix }}$ or (Belagenpumatucel-L) is a new specially tailored vaccine against advanced stage III/IV NSCLC. Lucanix ${ }^{\mathrm{na}}$ is a cellular vaccine collected from immortalised human lung cancer cell lines; H460, H520, SK-Lu-1 and transfected with EBV promoter/ antisense TGF $\beta 2$ which are further irradiated to maintain attenuation of the cell strains. The cells are harvested and injected subcutaneously in $0.4 \mathrm{ml}$ aliquots [42]. In a phase II study of Lucanix ${ }^{\text {tax }}$ which recruited 75 patients with a total of 550 injections, of the 61 assessable patients, $15 \%$ achieved a partial response. The 1 and 2 year survival rate was $68 \%$ and $52 \%$ respectively heralding a further phase III trial [43]. The multicentre phase III STOP trial started recruiting in 2008 with an intention to recruit 506 patients will finish sometime in late 2012. The trial is aimed to randomise two subgroups into a treatment group with vaccine and best supportive care while the placebo group receives best supportive care. The trial uses the same protocol of cellular preparation with once monthly injections for 18 months and then once at the $21^{\mathrm{st}}$ and $24^{\text {th }}$ month provided there is no toxicity or disease progression. The primary endpoint is overall survival (OS) while the secondary endpoints are progression free survival (PFS), quality of life (QOL) and time to progression between the vaccine and placebo. The results of this trial are keenly awaited and will definitely be a landmark vaccine trial for NSCLC and might change the way we treat these tumours indefinitely.

Listeria monocytogenes: Although Listeria Monocytogenes is a gram positive bacterium, one might wonder what is the relevance of a bacterium to immunotherapy? Listeria has some unique cellular and immunological properties. Listeria escapes the immune system and replicates intracellularly with up to $40 \%$ mortality. In contrast, another 
unique feature of this bacterium is its ability to mount an immune response that could potentially kill cancer cells. One of the mechanisms is its inherent intracytoplasmic nature and its direct stimulation of MHC class I CD8+ cells. In an experiment by Olino $\mathrm{K}$ et al. bioengineered Listeria cells expressing tumour associated antigens were able to mount an effective primary and memory T-cell response in murine models of colorectal liver metastasis, indicating that the use of Listeria is a possible option for cancer therapy [44]. The advantages of using Listeria or other bacterium is the fact that attenuating mutations are well documented and easily manipulated (1), the dose of the bacterium can be titrated (2), the response can be measured (3) and multiple sites could be used to inject the bacterium [45]. The use of L. Monocytogenes has been approached with trepidation but this unique bacterium could hold much promise for the future.

\section{The tumour environment and the balance between suppressor and effector cells}

Several investigators have been able to describe the fact that a tumour mass is a combination of tumour cells, the surrounding stroma with its fibroblastic reaction, released chemokines, immunosuppressive myeloid progenitor cells and regulatory $\mathrm{T}$ cells and in diametric contrast; immunostimulatory NKT and NK cells as well as gamma delta cells. Henceforth our discussion will focus on these particular factors and how modulating them can alter tumour prognosis and outcome.

The stromal reaction: The stromal reaction is a recent concept that has emerged that predicts survival in many tumours, particularly in colorectal carcinoma and pancreatic carcinoma. The concept has been revived after it was overlooked by so many investigators. The histological description of this reaction is that of myofibroblastic regeneration in response to tumour activation. The original description of this reaction and its significance in tumour progression was described by Desmouliere and colleagues [46] which is quite similar to a normal tissue injury and repair mechanism with the exception of persistence of myofibrolastic elements in tissue suggesting a local imbalance between survival and apoptosis of these cells. A recent analysis of hepatocellular carcinoma stromal reaction was able to identify specific gene signatures and compare them with other modes of classification (TNM and Barcelona Clinic Liver Cancer Stage) giving the study of stromal reactions further impetus [47]. Finally in a recent presentation, Mesker and colleagues, demonstrated the poor prognosis of a high stroma to tumour ratio in primary colorectal cancer giving pathologists another advantage in commenting and reporting these findings [48]. The significance of the stromal reaction is highlighted by the release of CCL2 and CXCL12 from the aforementioned myofibroblasts. These chemokines recruit Myeloid derived stem cells (MDSC) which become nitrated and nitrosylated and further suppress the recruitment of effector CD8+ cells. These MDSCs release TGF- $\beta$ and IL-10 creating a paracrine immunosuppressive environment as well. Thus the tumour

\begin{tabular}{|c|c|c|}
\hline $\mathrm{HSP}(\mathrm{kDa})$ & Location(s) & Significant function(s) \\
\hline $26-28$ & Cytosolic and nuclear & $\begin{array}{l}\text { Stabilisation of microfilaments and } \\
\text { cytokine signal transduction }\end{array}$ \\
\hline 60 & Mitochondrial & Protein assembly \\
\hline $70-74$ & $\begin{array}{l}\text { Cytosolic, nuclear, endoplasmic } \\
\text { and Mitochondrial }\end{array}$ & Protein folding and translocation \\
\hline 90 & $\begin{array}{l}\text { Cytosolic, nuclear and } \\
\text { endoplasmic }\end{array}$ & $\begin{array}{l}\text { Protein translocation and receptor } \\
\text { regulation }\end{array}$ \\
\hline $100-104$ & Cytosolic & Protein folding \\
\hline
\end{tabular}

Table 1: shows the heat shock proteins, their locations and their corresponding function stroma; contrary to what was known before is a dynamic entity that is just getting investigated more thoroughly.

Myeloid derived stem cells; MDSCs: Myeloid derived stem cells (MDSC) have been described just over 10 years ago. Research into this elusive heterogeneous subset of myeloid cells has raised many questions that have prompted immunologists to look further than the tumour cell. Human myeloid stem cells share a common myeloid surface antigen CD33 or broadly defined as $\mathrm{CD} 14^{\text {low }} \mathrm{CD} 11+$. On tissue sections MDSCs are defined by their high expression of inducible nitric oxide (iNOS) and arginase. Under normal circumstances MDSC are dormant cells that reside mainly in the bone marrow. Stimulation of MDSCs whether it is by infection, trauma or tumour by stem cell factor (SCF) promotes the migration of immature myeloid derived cells into the zone of injury coupled with several stimuli which include cytokines and chemokines. Myeloid derived stem cells have a unique immunosuppressive role in any tumour environment hence their maturation directly affects any therapeutic treatment modality. There are several factors that maintain the population of MDSCs in a tumour milieu. These include cyclooxygenase-2, IL-6, Granulocyte/macrophage-colony stimulating factor GM-CSF, SCF and Vascular endothelial growth factor VEGF. These agents finally converge on a JAK2/STAT3 signalling pathway which further promotes the expression of survival proteins -Survivin as well as anti-apoptotic proteins like BCL-XL, or the JAK3/STAT1/ STAT6 pathway which promotes the production of TGF- $\beta$, iNOS and arginase. The combined action of these cytokines and released proteins directly or indirectly influences Tumour infiltrating lymphocytes (TILs) and hence suppresses their cytotoxic function. Keeping all these points in perspective, a tumour destroying vaccine must tackle these cells in an equal par with tumours. Several modalities have been implicated in abrogating these cells.

(1) Inhibitors of cyclooxygenase-2 mainly aspirin and celecoxib are being thoroughly investigated as chemo-preventive modalities against adenocarcinomas of the colon and the breast.

(2) Alternatively, All Trans-retinoic acid (ATRA) promotes the dedicated maturation of MDSCs into DCs. In an experiment by Mirza et al, administration of ATRA in patients with Renal cell carcinoma resulted in the decrease of MDSC population [49].

(3) Another modality would be by reducing the MDSC population. Gemcitabine which is currently being used for NSCLC of the lung has the capability of eliminating MDSCs directly.

(4) Finally, another modality of intervention would be the blockade of maturation signals, like SCF and VEGF. In a recent large scale randomised trial- the VELOUR trial (Aflibercept Versus Placebo in Metastatic Colorectal Cancer after Failure of an Oxaliplatin Based Regimen) a novel scavenger antibody which binds to VEGF-A, VEGF-B and PGF, has been used on patients with refractory colorectal cancer who have already failed initial chemotherapy. The trial randomised 1200 patients to receive standard chemotherapy plus or minus Aflibercept (600 in each subgroup). The results were quite significant, with a doubling of the response to chemotherapy from $10 \%$ to $20 \%$ in the chemotherapy plus Aflibercept subgroup. The results demonstrated an increase in progression free survival by almost 45 days, but most significantly was the increase on overall survival from 12 months to 13.5 months. This discovery yet again proves that targeting a tumour is not a single modality of a peptide or protein vaccine but a combination of several important parameters that need to be dealt together.

Regulatory T cells (Tregs): Our discussion about cellular modalities of immunosuppression would not be complete without mentioning 
Regulatory T cells or Tregs. Tregs are currently described as CD4+/ CD25+/FOXP3+ T cells; initially described by Sakaguchi, Tregs play a key role in the maintenance of balance between autoimmunity and tumourigenesis such that its absence or attenuation results in a severe autoimmunity whereas its overwhelming presence in tumour milieu or peripheral circulation indicates a poor prognosis and a reduced survival [50]. The presence of high proportion of Tregs with/without CD8+ tumour infiltrating lymphocytes in a particular specimen has been implicated as a poor prognostic indicator in many tumours due to their immunosuppressive, antimitotic, antichemotactic and antiapoptotic effect that they exert on their neighbouring cells or new cells being recruited through chemokine signals released from the TILs. It is still not known what drives the maturity of these cell types to become dedicated Tregs or exactly how they come to reside next to tumours but radical excision of tumours as demonstrated from several studies certainly reduces their number and improves overall prognosis [51]. Henceforth, there have been several attempts to exactly quantify these cells with more exact markers such as CD127 [52]. Furthermore there have been efforts to preferentially delete these cells in an effort to enhance tumour killing. So far two chemotherapeutic agents have surfaced as potential modulators of Tregs; cyclophosphamide and gemcitabine. In a recent multicentre randomised trial on melanoma peptides on 167 patients, the outcome whether cyclophosphamide ameliorates the CD8 response was non-significant [53]. Another methodology which uses gemcitabine, has shown to transiently reduce the number of Tregs allowing a window of opportunity for vaccines [54]. Finally another novel modality fruitioned with the use of Denileukin difitox which is an engineered protein made from IL-2 and diphtheria toxin and thus preferentially binds to IL-2 receptor (IL-2R) which also happens to be highly elevated in Tregs. In a recent trial on mice use of denileukin difitox preferentially depleted the Tregs and made them more accessible for HER2/neu vaccine [55]. This elegant trial will certainly pave way for more trials and thus create a background on which Tregs are modulated until full vaccine therapy is implemented.

\section{The counterbalancing effector cells}

Gamma-delta T cells: Gamma delta T-cells are a unique subset of cells that have been investigated for the last five years for their potential in cellular immunotherapy and adoptive T-cell therapy. Traditional T-cells express $\alpha / \beta$ T-cell surface receptor that binds to MHCI or MHCII expressing APCs with an antigen loaded between the two grooves. However, unlike traditional $\alpha / \beta$ T-cells, $\gamma / \delta \mathrm{T}$ cells have a limited T-cell surface repertoire. For simplicity we will suffice with the discussion of the canonical $\mathrm{V} \gamma 9 \mathrm{~V} \delta 2$ cells which are mainly found in peripheral blood whereas intraepithelial $\gamma / \delta$ cells that line the gut are mainly $\mathrm{V} \delta 1$. Additionally, $\gamma \delta$-T cells do not recognise antigens with the help of MHCI or MHCII, instead they interact with stress induced MHC Like molecule called MICA or MICB expressed on tumour cells or cells infected with bacteria. Upon appropriate stimulation these cells are able to induce apoptosis as well as cell lysis. This has been attributed to direct ligand-ligand interaction or release of granzyme and perforin. Aminobiphosphonates like risedronate and zoledronic acid have been shown to be unique stimulants for these types of cells. Harvesting these cells, followed by their stimulation with zoledronic acid and Interleukin-2, has been a key methodology in using these cells as adoptive T-cell therapy in patients in Non Small Cell Lung Cancer [56]. Subsequent experiments with patients harbouring terminally advanced breast cancer has shown a $20 \%$ partial response rate and $10 \%$ stable disease in a group of 10 patients [57]. Another experiment conducted by Cabillic et al used DCs to pulse the $\gamma / \delta$ T cells and demonstrated a higher level of cytotoxicity [58]. These results accompanied by several other pilot experiments being conducted have shown that $\gamma / \delta \mathrm{T}$ cells demonstrate a reasonably reliable clinical outcome. However, further experiments have equally shown that the tumour inhibitory effect of these cells is further reliant on the cell surface receptors that they carry (Natural Killer Receptors) as well as the cytokine milieu that they are maturing in which could skew the immune response towards immunostimulatory or immunosuppressive [59,60]. Additionally, stimulation of $\gamma / \delta$ cell lines promotes the stimulation of NK cells carrying the NKG2D surface receptor CD137/CD137L which is crucial in clearing of MHC negative tumours [61]. Ultimately, we state that the use of $\gamma / \delta$ cells is in its infancy but evidence is accumulating for their potential use in the future protocols of cellular immunotherapy.

iNKT cells: Invariant Natural Killer $\mathrm{T}$ cells are another unique heterogenous subset of $\mathrm{T}$ cells that share common features with $\mathrm{T}$ cells (CD3+) as well as variable amount of Natural Killer cell features such as CD16 and CD56. Another unique characteristic of these cells is that they have a fixed TCR rearrangement similar to $\gamma / \delta$ T cells which gets activated by an MHC like glycoprotein on DCs or B cells called CD1d. Peripheral counts of iNKT cells vary considerably among species but are about $0.2 \%-0.5 \%$ in humans with substantially greater numbers in spleen, omentum and bone marrow. One of the earliest enthusiasms about this peculiar subset of cells was that it was able to directly lyse tumour cells through activation with a glycolipid molecule, alpha galactosyl ceramide in murine models. Further proof of their immune surveillance capabilities came from evidence collected from tissue specimens in patients with colorectal cancer as an independent marker of favourable prognosis [62]. However, in other solid tumour specimens like liver, even with infiltration of the tumour microenvironment with iNKT cells, they did not generate the same favourable prognostic parameters [63]. Further evidence demonstrated that iNKT cells have almost the same plasticity and variability with $\mathrm{CD} 4+\mathrm{T}$ cells and release the same variety of cytokines as Th1, Th2 and Th17 dedicated T cell lines which made these cells more difficult to work with. Recent citations have shown that native tumours secreting the chemokines CXCL16 which is another favourable prognostic indicator for colorectal cancer might shape the reciprocal iNKT response [64]. CXCR6 is the receptor for the ligand CXCL16 and happens to be expressed on CD4 ; low cytokine, non-lymphoid homing, and high granzyme producing cells which is another measure of complexity to these cells [65]. Early clinical trials using the T-cell ligand alpha-galactosyl ceramide(KRN7000) showed disappointing results when the initial dose was escalated 96 fold [66]. Henceforth, iNKT cells were approached with tepidity for the subsequent years. A more recent trial in patients with head and neck squamous cell carcinoma pulsed with KRN7000 showed a better response when DCs were used as primary stimulating cells as opposed to iNKT cells. In this modality, KRN7000 pulsed DCs were administered intranasally while the iNKT cells were infused into the tumour feeding vessels. Five patients demonstrated objective tumour regression while 7 of the original patients showed iNKT cell dependent regression in the tumour environment [67].

NK cells: Natural Killer cells are a subtype of cytotoxic lymphocytes that express CD16 and CD56 as surface markers but lack the pan-T cell CD3 marker. Natural killer cells have been named after the notion that they do not need stimulation to kill MHCI low or deficient cells. NK cell activity is delicately balanced between stimulatory and inhibitory signals (Table 2), FC-gamma-RIII, cytokines and recently stimulated DCs. In brief, NK cells have been known to kill tumour cells by "surveying" their respective surfaces for low levels of MHC expression, and hence direct cytotoxic killing by releasing perforin and granzyme. A similar mechanism is used to kill cells infected with viruses. It has 


\begin{tabular}{|c|c|c|}
\hline & NK Receptors & Corresponding Ligand/Cell Receptors \\
\hline \multirow{6}{*}{$\begin{array}{r}\frac{n}{0} \\
\frac{0}{3} \\
\frac{0}{\infty} \\
\frac{0}{\sigma} \\
\frac{0}{<}\end{array}$} & KIR2DL1 & HLA-C group 2 \\
\hline & KIR2DL2/3 & HLA-C group 1 \\
\hline & KIR3DL1 & HLA-Bw4 \\
\hline & KIR3DL2 & HLA-A3 and HLA -A11 \\
\hline & LIR-1 & HLA class I molecules \\
\hline & NKG2A & HLA-E \\
\hline \multirow{5}{*}{ 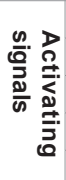 } & KIR2DS1 & HLA-C \\
\hline & NKG2D & ULBP-1-ULBP4, MICA and MICB \\
\hline & NKp46 & Viral heamagglutinin \\
\hline & CD16 & igG \\
\hline & BY55 & HLA-C \\
\hline
\end{tabular}

Table 2: NK cells harbour two sets of receptors; inhibitory and activating. Depending on the interaction of the ligand with the corresponding receptor, NK will signal the activating or inhibitory downstream cascade. In addition to the ligand/receptor interaction, debris from apoptotic and tumour cells, cytokines and costimulatory ligands as well dendritic cells can potentially skew the relevant signal, the interaction and hence the immune outcome.

been difficult to use NK cell in vaccine therapy; however, there is evidence that using NK cells in paediatric and adult haplo-identical allogenic transplants is effective in controlling minimal residual disease or imbalanced chimerism post haplo-stem cells transplant [68]. The experimental protocols that purely use NK cells as the sole modality of tumour therapy have not been as abundant but they are increasingly being studied in haematological malignancies [69]. On the other hand, NK cells are naturally used in conjunction with DCs in immunotherapy whereby both cell types engage in elaborate cross talk and hence NK cells assist DCs in tumour eradication. The mechanism of DC and NK cell interaction is explained in figure below.

\section{The Future}

Our discussion has not touched on every vaccine trial and pioneering methodology; however, the future holds promise for combinatorial approaches of vaccines in conjunction with immunomodulators. The latest success story of the latest lung vaccine trials has boosted the enthusiasm in cellular vaccines. The comparatively overwhelming success of monoclonal antibodies with respect to cellular vaccines or peptide vaccine components has prompted researcher to approach cellular vaccine with trepidation. Yet the complexity of cellular interactions has just recently given fruition to Aflibetacept, which once again simplifies the problem of cellular vaccines down to single bulleted agents. Another setback came a few months ago when the androgen suppressor Abiraterone has superseded Sipuleucel with at least 3 months. However, very recent evidence has shown that Sipuleucel is indeed useful for patients over the age of 70 which proves a concept that there is room for this treatment modality. These and several other failed active cellular vaccines have practically distanced several researchers from active cellular therapy and opted them for looking towards more simple options that give tangible results. Henceforth, the question raises itself, what is the problem with cellular vaccines? We can clearly quote that viral vaccine components have a predictable and resolute outcome; however, when it comes to tumour vaccines our immune system is indeed mounting a response but it is not enough in terms of tumour eradication or even tumour control. Thus although our understanding of interactions of DCs and T-cells has matured, there is a possibility that we have to vaccinate at risk patients like smokers, alcohol abusers, diabetics, or patients with colonic polyposis well before there is full manifestation of tumour(s). This might seem provocative but with ever increasing burden of tumours, there might be no opportunity other than to adopt radical methodologies and vaccinate the greatest number of at risk patients. Whether cellular vaccine biology will triumph over chemotherapy and monoclonal vaccine therapy is subject to successful trials and outcomes.

\section{Definition}

SEREX: Serological Analysis of Recombinant cDNA Expression libraries of human tumours with autologous serum. Is basically a technique aimed at finding autologous human sera against human tumours. For more information about SEREX: http://www. cancerimmunity.org/SEREX/introduction.htm

Cross Presentation: is the mechanism whereby extracellular antigens are presented on MHC I as opposed to MHC II and thus stimulate CD8+ T cells. Cross presentation is one of the unique pathways that allow the detection and mount an immune response against viruses and parenchymal mutations outside the bone marrow. The outcome of cross presentation, depending on the costimulation and other factors is either tolerance or immune response. Cross Presentation has important implications for vaccine subunit development.

CD137: is a member of the Tumour Necrosis Factor Receptor Superfamily (TNFRSF9) which binds to TNFSF9 expressed on Antigen Presenting Cells, Dendritic Cells and Activated B cells. The interaction acts as a protective mechanism for CD8+ cells from activation induced cell death which is crucial for maintaining tumour specific CD8 clones.

\section{References}

1. Palucka K, Banchereau J (2012) Cancer immunotherapy via dendritic cells. Nat Rev Cancer 12: 265-277.

2. Reichardt $P$, Dornbach $B$, Gunzer $M$ (2010) APC, T cells, and the immune synapse. Curr Top Microbiol Immunol 340: 229-249.

3. Huppa JB, Davis MM (2003) T-Cell-Antigen Recognition And The Immunological Synapse. Nat Rev Immunol 3: 973-983.

4. Pickering AM, Davies KJ (2012) Differential roles of proteasome and immunoproteasome regulators Pa28alphabeta, Pa28gamma and Pa200 in the degradation of oxidized proteins. Arch Biochem Biophys.

5. Compeer EB, Flinsenberg TW, van der Grein SG, Boes M (2012) Antigen processing and remodeling of the endosomal pathway: requirements for antigen cross-presentation. Front Immunol 3: 37.

6. Lattanzi L, Rozera C, Marescotti D, D’Agostino G, Santodonato L, et al. (2010) IFN-alpha boosts epitope cross-presentation by dendritic cells via modulation of proteasome activity. Immunobiology 216: 537-547.

7. Torigoe T, Tamura Y, Sato N (2009) Heat shock proteins and immunity: application of hyperthermia for immunomodulation. Int J Hyperthermia 25: 610 616.

8. Kettern N, Rogon C, Limmer A, Schild H, Höhfeld J (2011) The Hsc/Hsp70 co-chaperone network controls antigen aggregation and presentation during maturation of professional antigen presenting cells. PLoS One 6: e16398.

9. Choi DH, Woo JK, Choi Y, Seo HS, Kim CW (2011) A novel chimeric DNA vaccine: Enhancement of preventive and therapeutic efficacy of DNA vaccine by fusion of Mucin 1 to a heat shock protein 70 gene. Mol Med Report 4: 885890.

10. Kabakov AE, Kudriavtsev VA, Makarova luM (2011) Inhibitors of the heat shock protein 90 activity: a novel class of tumor radiosensitizers. Radiats Bio Radioecol 50: 528-535.

11. Uram JN, Black CM, Flynn E, Huang L, Armstrong TD, et al. (2011) Nondominant CD8 T cells are active players in the vaccine-induced antitumor immune response. J Immunol 186: 3847-3857

12. Cordaro TA, de Visser KE, Tirion FH, Schumacher TN, Kruisbeek AM (2002) Can the low-avidity self-specific $\mathrm{T}$ cell repertoire be exploited for tumor rejection? J Immunol 168: 651-660.

13. Miyara M, Sakaguchi S (2011) Human FoxP3(+)CD4(+) regulatory T cells: their knowns and unknowns. Immunol Cell Biol 89: 346-351.

14. Robert C, Thomas L, Bondarenko I, O'Day S, M D JW, et al. (2011) Ipilimumab 
Citation: Boghossian S, Von-Delwig A (2012) Tumour Vaccines, Monoclonals, Proteins or Whole Cell Therapies. J Vaccines Vaccin S1:003. doi:10.4172/2157-7560.S1-003

plus dacarbazine for previously untreated metastatic melanoma. N Engl J Med 364: 2517-2526.

15. Chung KY, Gore I, Fong L, Venook A, Beck SB, et al. (2010) Phase II study of the anti-cytotoxic T-lymphocyte-associated antigen 4 monoclonal antibody, tremelimumab, in patients with refractory metastatic colorectal cancer. J Clin Oncol 28: 3485-3490.

16. Rosenblatt J, Glotzbecker B, Mills H, Vasir B, Tzachanis D, et al. (2011) PD-1 blockade by CT-011, anti-PD-1 antibody, enhances ex vivo T-cell responses to autologous dendritic cell/myeloma fusion vaccine. J Immunother 34: 409-418.

17. Greiner JW, Zeytin H, Anver MR, Schlom J (2002) Vaccine-based therapy directed against carcinoembryonic antigen demonstrates antitumor activity on spontaneous intestinal tumors in the absence of autoimmunity. Cancer Res 62 : 6944-6951.

18. Ueda Y, Itoh T, Nukaya I, Kawashima I, Okugawa K, et al. (2004) Dendritic cellbased immunotherapy of cancer with carcinoembryonic antigen-derived, HLAA24-restricted CTL epitope: Clinical outcomes of 18 patients with metastatic gastrointestinal or lung adenocarcinomas. Int J Oncol 24: 909-917.

19. Gulley JL, Arlen PM, Tsang KY, Yokokawa J, Palena C, et al. (2008) Pilot study of vaccination with recombinant CEA-MUC-1-TRICOM poxviral-based vaccines in patients with metastatic carcinoma. Clin Cancer Res 14: 3060-3069.

20. Banchereau J, Ueno H, Dhodapkar M, Connolly J, Finholt JP, et al. (2005) Immune and clinical outcomes in patients with stage IV melanoma vaccinated with peptide-pulsed dendritic cells derived from CD34+ progenitors and activated with type I interferon. J Immunother 28: 505-516.

21. Luiten RM, Kueter EW, Mooi W, Gallee MP, Rankin EM, et al. (2005) Immunogenicity, including vitiligo, and feasibility of vaccination with autologous GM-CSF-transduced tumor cells in metastatic melanoma patients. J Clin Oncol 23: 8978-8991.

22. Hersey P, Halliday GM, Farrelly ML, DeSilva C, Lett M, et al. (2008) Phase I/II study of treatment with matured dendritic cells with or without low dose IL-2 in patients with disseminated melanoma. Cancer Immunol Immunother 57: 10391051.

23. Tyagi P, Mirakhur B (2009) MAGRIT: the largest-ever phase III lung cancer trial aims to establish a novel tumor-specific approach to therapy. Clin Lung Cancer 10: $371-374$

24. Zaretsky JZ, Barnea I, Aylon Y, Gorivodsky M, Wreschner DH, et al. (2006) MUC1 gene overexpressed in breast cancer: structure and transcriptional activity of the MUC1 promoter and role of estrogen receptor alpha (ERalpha) in regulation of the MUC1 gene expression. Mol Cancer 5: 57.

25. Ninkovic T, Kinarsky L, Engelmann K, Pisarev V, Sherman S, et al. (2009) Identification of O-glycosylated decapeptides within the MUC1 repeat domain as potential MHC class I (A2) binding epitopes. Mol Immunol 47: 131-140.

26. Ramlau R, Quoix E, Rolski J, Pless M, Lena H, et al. (2008) A phase II study of Tg4010 (Mva-Muc1-II2) in association with chemotherapy in patients with stage III/IV Non-small cell lung cancer. J Thorac Oncol 3: 735-744.

27. Butts C, Murray RN, Smith CJ, Ellis PM, Jasas K, et al. (2010) A multicenter open-label study to assess the safety of a new formulation of BLP25 liposome vaccine in patients with unresectable stage III non-small-cell lung cancer. Clin Lung Cancer 11: 391-395.

28. Zhang YF, Tang XD, Gao JH, Fang DC, Yang SM (2011) Heparanase: a universal immunotherapeutic target in human cancers. Drug Discov Today 16: 412-417

29. Zhang L, Sullivan PS, Goodman JC, Gunaratne PH, Marchetti D (2011) MicroRNA-1258 suppresses breast cancer brain metastasis by targeting heparanase. Cancer Res 71: 645-654.

30. Wang GZ, Tang XD, Lu MH, Gao JH, Liang GP, et al. (2011) Multiple Antigenic Peptides of Human Heparanase Elicit a Much More Potent Immune Response against Tumors. Cancer Prev Res (Phila) 4: 1285-1295.

31. Idenoue S, Hirohashi Y, Torigoe T, Sato Y, Tamura Y, et al. (2005) A potent immunogenic general cancer vaccine that targets survivin, an inhibitor of apoptosis proteins. Clin Cancer Res 11: 1474-1482.

32. Wobser M, Keikavoussi $P$, Kunzmann $V$, Weininger $M$, Andersen $M H$, et al (2006) Complete remission of liver metastasis of pancreatic cancer under vaccination with a HLA-A2 restricted peptide derived from the universal tumor antigen survivin. Cancer Immunol Immunother 55: 1294-1298.
33. Weide B, Pascolo S, Scheel B, Derhovanessian E, Pflugfelder A, et al (2009) Direct injection of protamine-protected mRNA: results of a phase $1 / 2$ vaccination trial in metastatic melanoma patients. J Immunother 32: 498-507.

34. Trepiakas R, Berntsen A, Hadrup SR, Bjørn J, Geertsen PF, et al. (2010) Vaccination with autologous dendritic cells pulsed with multiple tumor antigens for treatment of patients with malignant melanoma: results from a phase I/II trial. Cytotherapy 12: 721-734.

35. Snook AE, Eisenlohr LC, Rothstein JL, Waldman SA (2007) Cancer mucosa antigens as a novel immunotherapeutic class of tumor-associated antigen. Clin Pharmacol Ther 82: 734-739

36. Snook AE, Stafford BJ, Li P, Tan G, Huang L, et al. (2008) Guanylyl cyclase $\mathrm{C}$-induced immunotherapeutic responses opposing tumor metastases without autoimmunity. J Natl Cancer Inst 100: 950-961.

37. Hishinuma M, Ohashi KI, Yamauchi N, Kashima T, Uozaki H, et al. (2006) Hepatocellular oncofetal protein, glypican 3 is a sensitive marker for alphafetoprotein-producing gastric carcinoma. Histopathology 49: 479-486.

38. Yamauchi N, Watanabe A, Hishinuma M, Ohashi K, Midorikawa $Y$, et al (2005) The glypican 3 oncofetal protein is a promising diagnostic marker for hepatocellular carcinoma. Mod Pathol 18: 1591-1598.

39. Yoshikawa T, Nakatsugawa M, Suzuki S, Shirakawa H, Nobuoka D, et al (2011) HLA-A2-restricted glypican-3 peptide-specific CTL clones induced by peptide vaccine show high avidity and antigen-specific killing activity against tumor cells. Cancer Sci 102: 918-925.

40. Plosker GL (2011) Sipuleucel-T in metastatic castration-resistant prostate cancerdagger: profile report. BioDrugs 25: 255-256.

41. Garcia JA (2011) Sipuleucel-T in patients with metastatic castration-resistan prostate cancer: an insight for oncologists. Ther Adv Med Oncol 3: 101-108.

42. Nemunaitis J, Dillman RO, Schwarzenberger PO, Senzer N, Cunningham C, et al. (2006) Phase II study of belagenpumatucel-L, a transforming growth factor beta-2 antisense gene-modified allogeneic tumor cell vaccine in non-small-cell lung cancer. J Clin Oncol 24: 4721-4730.

43. Nemunaitis J, Nemunaitis M, Senzer N, Snitz P, Bedell C, et al. (2009) Phase I trial of Belagenpumatucel-L, a TGF-beta2 antisense gene modified allogeneic tumor vaccine in advanced non small cell lung cancer (NSCLC) patients. Cancer Gene Ther 16: 620-624

44. Olino K, Wada S, Edil BH, Pan X, Meckel K, et al. (2011) Tumor-Associated Antigen Expressing Listeria monocytogenes Induces Effective Primary and Memory T-Cell Responses Against Hepatic Colorectal Cancer Metastases. Ann Surg Oncol.

45. Carleton HA (2010) Pathogenic bacteria as vaccine vectors: teaching old bugs new tricks. Yale J Biol Med 83: 217-222.

46. Desmoulière A, Guyot C, Gabbiani G (2004) The stroma reaction myofibroblast a key player in the control of tumor cell behavior. Int J Dev Biol 48: 509-517.

47. Gao Q, Wang XY, Qiu SJ, Zhou J, Shi YH, et al. (2011) Tumor stroma reactionrelated gene signature predicts clinical outcome in human hepatocellular carcinoma. Cancer Sci 102: 1522-1531.

48. Mesker WE, Junggeburt JM, Szuhai K, de Heer P, Morreau H, et al. (2007) The carcinoma-stromal ratio of colon carcinoma is an independent factor for survival compared to lymph node status and tumor stage. Cell Oncol 29: 387398.

49. Mirza N, Fishman M, Fricke I, Dunn M, Neuger AM, et al. (2006) All-transretinoic acid improves differentiation of myeloid cells and immune response in cancer patients. Cancer Res 66: 9299-9307.

50. Elkord E, Alcantar-Orozco EM, Dovedi SJ, Tran DQ, Hawkins RE, et al. (2010) $T$ regulatory cells in cancer: recent advances and therapeutic potential. Expert Opin Biol Ther 10: 1573-1586.

51. Sellitto A, Galizia G, De Fanis U, Lieto E, Zamboli A, et al. (2011) Behavior of Circulating CD4+CD25+Foxp3+ Regulatory T Cells in Colon Cancer Patients Undergoing Surgery. J Clin Immunol 31: 1095-1104.

52. Beyer M, Classen S, Endl E, Kochanek M, Weihrauch MR, et al. (2011) Comparative Approach to Define Increased Regulatory T Cells in Different Cancer Subtypes by Combined Assessment of CD127 and FOXP3. Clin Dev Immunol 2011: 734036.

53. Slingluff CL Jr, Petroni GR, Chianese-Bullock KA, Smolkin ME, Ross MI, et al. (2011) Randomized multicenter trial of the effects of melanoma-associated 
Citation: Boghossian S, Von-Delwig A (2012) Tumour Vaccines, Monoclonals, Proteins or Whole Cell Therapies. J Vaccines Vaccin S1:003. doi:10.4172/2157-7560.S1-003

helper peptides and cyclophosphamide on the immunogenicity of a multipeptide melanoma vaccine. J Clin Oncol 29: 2924-2932.

54. Rettig L, Seidenberg S, Parvanova I, Samaras P, Curioni A, et al. (2011) Gemcitabine depletes regulatory T-cells in human and mice and enhances triggering of vaccine-specific cytotoxic T-cells. Int J Cancer 129: 832-838.

55. Gritzapis AD, Voutsas IF, Baxevanis CN (2011) Ontak reduces the immunosuppressive tumor environment and enhances successful therapeutic vaccination in HER-2/neu-tolerant mice. Cancer Immunol Immunother 61: 397 407

56. Nakajima J, Murakawa T, Fukami T, Goto S, Kaneko T, et al. (2010) A phase I study of adoptive immunotherapy for recurrent non-small-cell lung cancer patients with autologous gammadelta T cells. Eur J Cardiothorac Surg 37: 1191-1197.

57. Meraviglia S, Eberl M, Vermijlen D, Todaro M, Buccheri S, et al. (2010) In vivo manipulation of Vgamma9Vdelta2 $\mathrm{T}$ cells with zoledronate and low-dose interleukin-2 for immunotherapy of advanced breast cancer patients. Clin Exp Immunol 161: 290-297.

58. Cabillic F, Toutirais O, Lavoué V, de La Pintière CT, Daniel P, et al. (2010) Aminobisphosphonate-pretreated dendritic cells trigger successful Vgamma9Vdelta2 T cell amplification for immunotherapy in advanced cancer patients. Cancer Immunol Immunother 59: 1611-1619.

59. Silva-Santos B (2010) Promoting angiogenesis within the tumor microenvironment: the secret life of murine lymphoid IL-17-producing gammadelta T cells. Eur J Immunol 40: 1873-1876.

60. Angelini DF, Zambello R, Galandrini R, Diamantini A, Placido R, et al. (2010) NKG2A inhibits NKG2C effector functions of gammadelta T cells: implications in health and disease. J Leukoc Biol 89: 75-84

61. Maniar A, Zhang X, Lin W, Gastman BR, Pauza CD, et al. (2010) Human gammadelta $\mathrm{T}$ lymphocytes induce robust NK cell-mediated antitumo cytotoxicity through CD137 engagement. Blood 116: 1726-1733.

62. Tachibana T, Onodera H, Tsuruyama T, Mori A, Nagayama S, et al. (2005) Increased Intratumor Valpha24-positive Natural Killer T cells: A prognostic Factor for Primary Colorectal Carcinomas. Clin Cancer Res 11: 7322-7327.

63. Bricard G, Cesson V, Devevre E, Bouzourene H, Barbey C, et al. (2009) Enrichment of human CD4+ V(alpha)24/Vbeta11 invariant NKT cells in intrahepatic malignant tumors. J Immunol 182: 5140-5151.

64. Hojo S, Koizumi K, Tsuneyama K, Arita Y, Cui Z, et al. (2007) High-leve expression of chemokine CXCL16 by tumor cells correlates with a good prognosis and increased tumor-infiltrating lymphocytes in colorectal cancer Cancer Res 67: 4725-4731.

65. Kim $\mathrm{CH}$, Johnston $\mathrm{B}$, Butcher EC (2002) Trafficking machinery of NKT cells: shared and differential chemokine receptor expression among $V$ alpha 24(+) $\mathrm{V}$ beta $11(+)$ NKT cell subsets with distinct cytokine-producing capacity. Blood 100: $11-16$.

66. Giaccone G, Punt CJ, Ando Y, Ruijter R, Nishi N, et al. (2002) A phase I study of the natural killer T-cell ligand alpha-galactosylceramide (KRN7000) in patients with solid tumors. Clin Cancer Res 8: 3702-3709.

67. Yamasaki K, Horiguchi S, Kurosaki M, Kunii N, Nagato K, et al. (2010) Induction of NKT cell-specific immune responses in cancer tissues after NKT cell-targeted adoptive immunotherapy. Clin Immunol 138: 255-265.

68. Barrett AJ, Le Blanc K (2010) Immunotherapy prospects for acute myeloid leukaemia. Clin Exp Immunol 161: 223-232.

69. Curti A, Ruggeri L, D’Addio A, Bontadini A, Dan E, et al. (2011) Successfu transfer of alloreactive haploidentical KIR ligand-mismatched natural killer cells after infusion in elderly high risk acute myeloid leukemia patients. Blood 118 3273-3279.
This article was originally published in a special issue, Tumor Immunology Vaccines handled by Editor(s). Dr. Robert John Amato, The University of Texas, USA 